\title{
Uma geografia temporal do encontro
}

\author{
Vinicius Netto \\ Universidade Federal Fluminense, Rua Miguel de Frias 9, 24220-900 Niterói, RJ, \\ Brasil. E-mail: vmnetto@id.uff.br \\ João Vitor Meirelles \\ Laboratory on Human-Environment Relations in Urban Systems, Ecole Polytechnique \\ Federale de Lausanne, GR C1 455 (Bâtiment GR) - Station 2, CH-1015 Lausanne, \\ Suíça. E-mail: joao.meirelles@epfl.ch
}

\author{
Maíra Pinheiro \\ Escola Nacional de Ciência Estatística, Rua André Cavalcanti 106, Bairro de Fátima, \\ 20231-050 Rio de Janeiro, RJ, Brasil. E-mail: mairasoares.arq@ hotmail.com \\ Henrique Lorea \\ Universidade Federal do Rio Grande do Sul, Avenida Paulo Gama 110, Bairro \\ Farroupilha, 90040-060 Porto Alegre, RS, Brasil. \\ E-mail: henriquelorealeite@gmail.com
}

Artigo revisto recebido a 7 de Novembro de 2017

\begin{abstract}
Resumo. A integração de redes sociais e espaciais é fundamental para novas abordagens a cidades como sistemas de interacção. Neste artigo, propomos uma maneira de analisar as condições espaciais e temporais do encontro como condição da formação de redes sociais. Reunindo abordagens clássicas como a geografia temporal de Hägerstrand e o conceito de segregação como 'restrição de contato' de Freeman, e explorações recentes de dados de localização via mídia digital, analisamos a estrutura espaço-temporal de encontros potenciais nas trajetórias urbanas de usuários do Twitter diferenciados por níveis de renda no Rio de Janeiro. Esta abordagem permite estimar as posições dos usuários, visualizar grupos de renda e suas trajetórias no espaço urbano, identificar espaços de encontro potencial e os níveis de diversidade e segregação nos espaços públicos. O artigo conclui com uma discussão dos achados empíricos e a utilidade desta 'geografia temporal dos encontros' potenciais na cidade, possivel a partir da introdução de novas tecnologias de comunicação digital móvel.
\end{abstract}

Palavras-chave: redes sociais, encontro, segregação, mobilidade, dados de localização

Introdução: ligando a cidade social à cidade física

'Ligar a cidade física à cidade social é o desafio de nossos tempos' (Batty, 2017, p. 1; traduções dos autores).

'Como Glaeser e Jacobs antes dele argumentaram tão persuasivamente, cidades existem para gerar 'conexões'... Os vários processos que ocorrem nas cidades e aproximam as pessoas para produzir e trocar bens e ideias, definem uma multiplicidade de redes que permitem que as populações produzam materiais e informações para apoiar tal esforço' (Batty, 2013).

Cidades são redes sociais de pessoas e 
instituições cuja organização física permite a troca de artefatos e informações. Se ligar a 'cidade social' à 'cidade física' é um grande desafio nos estudos urbanos, como afirmam Batty (2013) e Hillier e Vaughan (2007), o problema de relacionar 'redes sociais' com o ambiente construído enquanto 'redes espaciais' está no seu centro. Em outras palavras, se quisermos avançar nossa compreensão da relação entre cidade e sociedade, precisamos nos aproximar dos elementos fundamentais dessa relação: a cidade como 'conjuntos de ações, interações e transações... padrões de fluxos, de redes de relações, pertencentes tanto a material físico, bem como os movimentos elusivos' (Batty, 2013, p. 9). Sabemos que redes sociais são formadas através de oportunidades de contato, encontro e interação (Freeman, 1978) como eventos sociais no tempo e no espaço. A cidade teve historicamente o papel de produzir tais eventos. Propomos abordar neste artigo quais são exatamente as condições materiais para a 'formação' dessas redes: as oportunidades do encontro no espaço e tempo.

Para tanto, vamos examinar as trajetórias no espaço urbano de pessoas diferenciadas por renda. Próxima a uma tendência recente em estudos focados no posicionamento dos agentes no espaço, nossa abordagem explora formas em que os encontros potenciais são moldados pelas trajetórias dos corpos no espaço urbano. Em outras palavras, vemos um grande potencial na análise do papel das trajetórias de diferentes agentes em uma cidade como meio para compreender os padrões de encontro como as circunstâncias que permitem a formação das redes sociais. É claro que esse objetivo envolve entrar em um tecido bastante elusivo dos nossos movimentos e encontros. Nossa abordagem inclui um método para capturar esse tecido, derivando ideias desde Hunt e Walker (1974) e Freeman (1978) sobre a segregação como 'restrições sobre contatos' à geografia temporal dos trajetos, de Hägerstrand (1970), à ideia de 'segregação em tempo real' e redes segregadas atuando na cidade (Netto et al., 2015; Netto e Krafta, 1999, 2001). Temos como objetivos:

i) explorar o papel das 'trajetórias' urbanas na criação de oportunidades de encontro, o papel do 'encontro' na formação das redes sociais - e seu oposto, na disjunção dos encontros na segregação;

ii) explorar o uso metodológico dos dados de localização das redes sociais digitais para compreender as trajetórias de usuários e inferir encontros potenciais entre eles;

iii) aplicar esta estrutura em um estudo empírico de redes de trajetórias e encontros de pessoas com diferentes níveis de renda no Rio de Janeiro, a fim de avaliar níveis de segregação em 'tempo real' e de diversidade social nas ruas; $\mathrm{e}$

iv) avaliar como os encontros são produzidos entre pessoas com diferentes níveis de renda, através de uma análise de redes de proximidade baseada em encontros potenciais.

Comecemos por propor um conceito de redes socioespaciais capaz de representar trajetórias e encontros no tempo e no espaço.

\section{O papel dos encontros na formação de redes sociais}

Como as cidades podem ser parte do modo como conhecemos outras pessoas e formamos redes sociais? Uma visão recente estabelece com rigor essa leitura ao reconhecer cidades como uma espécie de equilíbrio flutuante entre a 'densidade' de pessoas e sua 'conectividade' (Bettencourt, 2013). A comunicação e a conectividade entre as pessoas são altamente dependentes dos encontros, que por sua vez são uma função da 'distância' (Allen, 1977) e da 'mobilidade' dos atores. Encontros podem ser dispersos nas ruas ou polarizados em locais de trabalho, lazer ou consumo, em pontos de ônibus, estações de metrô, edifícios institucionais e assim por diante. Todos esses fatores podem ter impacto sobre nossos encontros, como faíscas de uma densa rede de movimentos a partir de locais residenciais.

Naturalmente, copresença e encontro podem implicar coisas diferentes em diferentes contextos - desde possibilidades abertas de interação até rejeição e medo. Seguindo o trabalho de Goffman (1961), Giddens (1984) e Hillier e Hanson (1984), entendemos a 'copresença' como corpos posicionados dentro de um campo onde podemos perceber a presença de outra pessoa (através da visão ou outros sentidos). Por sua 
vez, o 'encontro' pode ser definido como estarmos copresentes dentro de uma distância onde a interação se torna possível. Por sua vez, 'interação' significa envolver-se em troca comunicativa por gesto ou comunicação verbal. Como matéria-prima da vida social, a importância do encontro dificilmente pode ser exagerada. No entanto, não desejamos abordar a passagem do 'encontro' à 'interação', dado que isso exigiria observações de pessoas em suas trocas reais, algo que está fora do nosso interesse substantivo neste artigo.

Se o movimento deixasse traços visíveis, sua trama poderia revelar oportunidades de encontros que se desdobram no tempo e no espaço. Mapear essa trama é um dos objetivos deste artigo. Isso soa ambicioso, e só vem se tornando possível graças a novas tecnologias da informação e comunicação móvel, como veremos. Mas a ideia de mapear as trajetórias das pessoas no espaço e no tempo está longe de ser nova. O trabalho do geógrafo sueco Torsten Hägerstrand foi a primeira tentativa sistemática de captar trajetórias e entender as restrições que afetam nossas ações, no que ele chamou 'geografia temporal'. A abordagem de Hägerstrand (1970), popular no início dos anos 1980, vem ganhado novamente atenção com o novo foco em abordagens espaciais e temporais (Kwan, 2013; Lee e Kwan, 2011; Netto e Krafta, 1999, 2001; Park e Kwan, 2017), fazendo uso de tecnologias capazes de registrar $o$ movimento de atores (Gonzáles et al., 2008). Propomos neste artigo adicionar novas camadas a esta ideia, avaliando 'como as trajetórias das pessoas moldam suas oportunidades de encontro'. Essas trajetórias são traços elusivos de nossa presença no espaço. Se pudéssemos capturar pelo menos parte dessa trama, poderíamos ter uma ideia dos lugares e momentos do encontro - como as pessoas e grupos sociais materializam seus potenciais de interação.

Para reconhecer o potencial social do encontro, gostaríamos de explorar uma definição alternativa de 'redes sociais'. O termo apareceu no cotidiano nos anos 2000, associado com mídias de comunicação digital - mas é muito anterior a isso. Ele é usado desde os anos 1950 em uma área chamada Social Network Analysis (SNA), focada na formação de grupos sociais a partir de tipos de vinculação - digamos, por compartilharem interesses. A tradição em SNA se concentra na análise de redes variando de relações de poder à difusão de informação, epidemias em larga escala e a conectividade em small worlds ou pequenos mundos. Ela usa a teoria dos grafos para criar representações livres de espaço e tempo - um espaço abstrato, sem dimensões físicas, de pura topologia. Atores são representados por nós ou vértices e relacionamentos por ligações (Figura 1).

Não utilizaremos nesta etapa o conceito usual em SNA da rede como arranjo de atores e suas ligações. Ao invés dela, utilizaremos uma definição de rede social como um conjunto aberto de pessoas com contatos criados em circunstâncias de tempoespaço. Graficamente, não representamos atores por vértices e relacionamentos por ligações, como na Figura 1. Em vez disso, invertemos esta representação, vendo os atores como 'linhas de vida'. Assim, poderemos incluir o fator elementar do tempo. O encontro entre duas pessoas é representado pela 'intersecção' das suas linhas de vida. Os encontros são os nós. Esta representação não usual oferece a possibilidade de 'homologia' com a dimensão espacial do fenômeno, na qual as 'linhas de vida correspondem a trajetórias urbanas', e as circunstâncias do encontro correspondem às posições espaciais das convergências das linhas. Lugares de convergência de trajetórias são lugares de potencial encontro e conexão. Esta ideia busca agregar a dimensão temporal à espacial, ambas como 'inerentes' à rede social, algo que simplesmente não ocorre na representação usual de redes sociais. Esta representação torna a espacialidade e a temporalidade da rede social intuitivas, permitindo explorar o encontro como um fator-chave na formação de redes sociais (Figura 2).

Uma vez que tenhamos um mapa das linhas de vida dos atores como trajetórias urbanas, abandonaremos a representação estática e exclusivamente social das redes. Estamos tratando de redes sociais e espaciotemporais. Esta abordagem pode ser especialmente útil para detectar diferentes potenciais de encontro que possam conduzir a uma maior interação entre pessoas ou a uma falta sistemática de contato - uma forma 


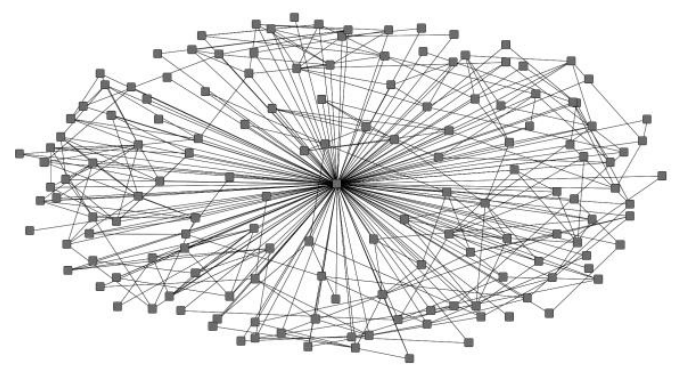

Figura 1. Rede social de relações pessoais centrada no 'ego' (fonte: Mirella Furtado).
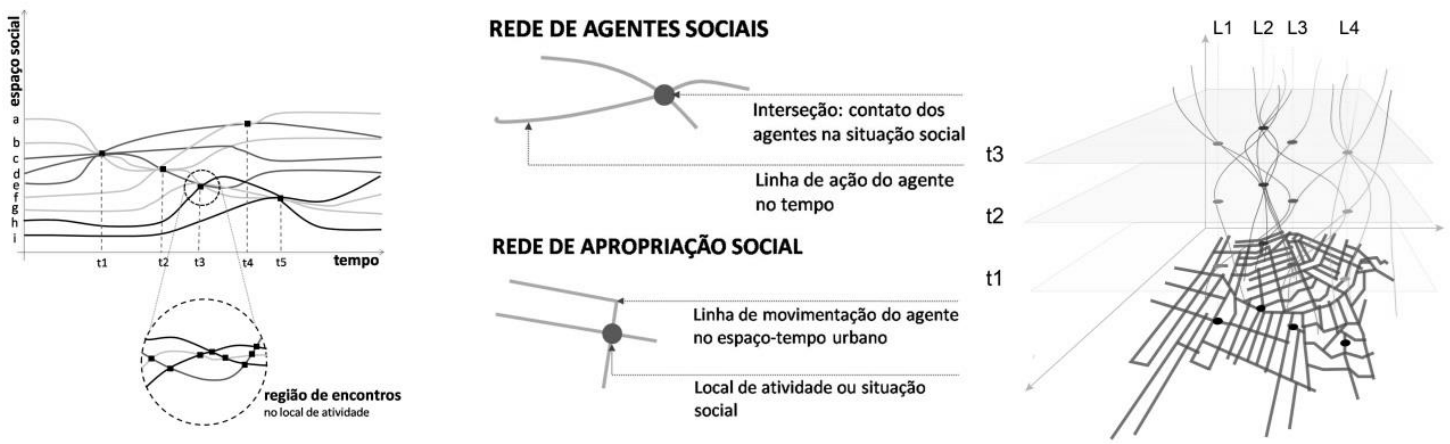

Figura 2. A homologia entre redes sociais no tempo (esquerda) e sua tradução no espaço (centro). À direita, temos a representação das redes sociais no espaço-tempo.

sutil e penetrante de 'segregação em tempo real' na vida urbana. Vejamos como esse poderia ser o caso.

\section{Trajetórias e contato potencial entre grupos socialmente diferentes}

Uma visão inovadora do papel social dos encontros foi proposta pelo teórico de redes sociais Linton Freeman: 'Todas as restrições à interação, envolvendo o espaço físico ou não, são formas de segregação - no espaço social' (Freeman, 1978, p. 413). O conceito de Freeman sugere que a ausência de encontros entre pessoas pode gerar uma forma sutil mas eficaz de segregação, especialmente entre pessoas socialmente diferentes.

Este foco na segregação baseada em pessoas e não em lugares é uma tendência recente nos estudos de segregação (Kwan, 2013; Lee e Kwan, 2011; Netto e Krafta, 1999, 2001; Netto et al., 2015; Park e Kwan, 2017; Schnell e Yoav, 2001, 2005; Selim,
2015; Wissinsk et al., 2016). Essa possibilidade oferece uma perspectiva nova sobre o delicado tecido de encontros que mantêm os sistemas sociais locais integrados. As condições de encontro e interação são moldadas pela 'diferença social', incluindo, entre outras coisas, estilos de vida influenciados pelas possibilidades comportamentais e pelos interesses oferecidos por diferentes níveis de renda. As diferenças sociais determinam compatibilidades e incompatibilidades, proximidades e distâncias e moldam a probabilidade de as pessoas se constituírem como grupos práticos (Bourdieu, 1985). Inversamente, as diferenças podem dificultar a aproximação entre os agentes. As características dos agentes desempenham um papel ativo na geração de situações de encontro.

Qual é a chance de conhecer alguém de um grupo social diferente? O mapeamento do movimento pode nos ajudar a entender $\mathrm{o}$ surgimento de situações de encontro entre grupos sociais. Por exemplo, certas áreas de 
uma cidade (uma rua movimentada, o centro ou uma centralidade local), bem servidas por transporte, podem atrair pessoas com diferentes níveis de renda. Lugares de atividade podem aumentar o potencial de encontros entre aqueles que compartilham interesses e mobilidades semelhantes. A renda tem um papel neste processo. Pessoas com orçamentos menores enfrentam mais restrições na mobilidade. Os dados empíricos sobre despesas de transporte no Brasil mostram que os grupos de maior renda não só gastam mais do que grupos de baixa renda, eles o fazem mais do que proporcionalmente (POF, 2009). Por sua vez, as limitações na mobilidade aumentam o 'localismo', a dependência da proximidade para produzir relações sociais estáveis (Fischer e Shavit, 1995). Nesses casos, as pessoas tendem a usar lugares na vizinhança para criar e manter relacionamentos. Outros estudos empíricos mostraram que a segregação residencial, níveis mais elevados de homofilia (similaridade nas redes sociais) e diferentes graus de conectividade nas redes pessoais têm correlações consistentes com diferenças de renda (Marques, 2012).

Por sua vez, as semelhanças nos 'padrões de mobilidade e apropriação do espaço' podem levar ao aumento da densidade de encontros entre pessoas socialmente similares. A renda, a localização residencial e os trajetos das pessoas podem estar associados em um círculo que leva a aumentos ou diminuições sistemáticas no potencial para criar, manter e expandir redes sociais. Mas como podemos verificar se esse é ou não o caso? Como ver onde o potencial de encontro entre os diferentes se materializa?

Para responder a estas perguntas, precisamos examinar as trajetórias de pessoas socialmente diferentes e onde se sobrepõem. Estes lugares de sobreposição gerariam as oportunidades do encontro. Mesmo que não pensemos nisso, nossas trajetórias diárias constituem a espinha dorsal dos nossos encontros e vida social. Por um lado, pobres e ricos podem viver distantes entre si, espacialmente segregados, mas eles se movem e podem até mesmo compartilhar espaços públicos. Por outro lado, a distância, associada a diferenças de renda, mobilidade e estilo de vida, pode trazer desigualdades na capacidade de acesso a determinados lugares e dificuldades para participarmos de situações sociais. Diferenças e incompatibilidades em padrões de movimento são formas de 'disjunção de encontros' (Netto, 2014) - uma maneira de diluir a possibilidade de contatos que de outra forma poderiam acontecer. A disjunção dos encontros pode ser mais ativa entre pessoas socialmente diferentes. Simplificando, haveria uma maior chance de encontrar e criar redes entre pessoas que compartilham níveis de renda semelhantes.

Em estudos anteriores (Netto et al., 2015; Netto e Furtado, 2017), propusemos uma medida de mobilidade individual. Consideramos a dimensão fractal dos caminhos percorridos por centenas de entrevistados e o número de atividades realizadas por eles ao longo do dia, ambos sujeitos a formas de transporte e níveis de renda. A medida foi capaz de detetar diferenças significativas: quanto maior a renda, maior a mobilidade. Entretanto, essa mobilidade seria capturada de modo frágil em um estudo utilizando dados do Twitter, que acaba se limitando a colher trajetos apenas entre as localizações dos tweets postados, como veremos. O recurso ao Twitter permite acessar trajetos parciais de milhares de pessoas, mas sem acesso a modos de transporte e outras informações. Mas se não temos condição de avaliar a mobilidade, ainda temos condição de verificar as trajetórias parciais de atores socialmente diferentes. Podemos examinar portanto os graus de 'segregação' nesses trajetos, e as 'possibilidades de encontro' entre pessoas socialmente distintas.

Uma hipótese surge dessa leitura: a probabilidade de encontros inclui, mas vai 'além' da localização e segregação residencial. Ela seria moldada pela renda, pela distribuição de atividades ao longo das ruas como canais de acessibilidade, e pelos diferentes caminhos das pessoas. Essas ideias começam a retratar o tecido elusivo dos encontros em uma cidade, uma trama impregnada de formas sutis de 'segregação em tempo real' expressas em trajetórias diárias. Mas como podemos chegar a uma 'geografia dos encontros' no tempo? 


\section{O uso de dados de localização do Twitter}

A ideia de visualizar os fluxos tremendamente complexos da população de uma cidade soava, até recentemente, quase impossível. Essa dificuldade começou a mudar com a introdução das redes digitais e tecnologias de comunicação pessoal capazes de registrar o movimento de um grande número de pessoas. A partir daí, uma série de novos trabalhos vêm utilizando dados de localização de usuários de mídia social, a fim de extrair informações de padrões humanos de movimento. Por exemplo, Lee et al. (2011) verificaram como a utilização dos canais de comunicação móveis de informação afeta opções de consumo just in time no comportamento de viagem. Li et al. (2011) e Ribeiro et al. (2012) desenvolveram formas para inferir a localização de geotags do Twitter, enquanto Veloso e Ferraz (2011) e Takhteyev et al. (2012) inferiram informações através de modelos de regressão correlacionando frequências dos tweets com eventos do mundo real, enquanto Boettcher e Lee (2012) aplicaram técnicas de aglomeração espacial (clustering) baseadas em densidade.

No espírito desses trabalhos, realizamos um estudo empírico na cidade do Rio de Janeiro fazendo uso de metadados do Twitter, rede digital de troca de informações. O Twitter oferece possibilidades particularmente atraentes, por tornar seu banco público seguindo um princípio de anonimato. $\mathrm{O}$ conjunto de variáveis fornecidas pelo Twitter API inclui IDs de usuário, juntamente com uma 'assinatura' espaço-temporal: o momento e as coordenadas geográficas (geostamp) de cada tweet postado por usuários que optam por ter a localização GPS em seus celulares ligados. Isso gera a possibilidade de inferir características do comportamento espacial a nível individual, envolvendo grandes amostras. Naturalmente, riscos de generalização do comportamento a partir de usuários individuais devem ser cuidadosamente tidos em conta (Longley et al., 2015). Este estudo é intencionado como uma proxy do cenário real de trajetórias de atores socialmente diferenciados. Abordamos o problema em uma sequência de passos.
No primeiro passo, coletamos metadados de tweets com posições espaço-temporais postados no Rio através do streaming API oficial do Twitter entre 12 de novembro (00:07:13) e 14 (02:36:45) de 2014, durante um período de 56 horas, usando um script criado na linguagem python, gerando um banco de dados de 20.192 usuários e 333.407 tweets. Devido a limites computacionais considerando novos procedimentos, como a geração de caminhos mais curtos entre as posições de tweet, optamos por trabalhar com esta amostra. Em seguida, verificamos se a amostra tinha representatividade quanto ao comportamento e localização de usuários em um banco de dados de 241 horas, com 70.403 usuários e 2.252.348 tweets registrados ao longo de 18 dias. Encontramos uma correlação linear de Pearson de 0,976 (pvalor $=2,2 \mathrm{e}-16$ ) entre os conjuntos de dados quanto a distribuição espacial do tweets de acordo com setores censitários (Censo 2010, Instituto Brasileiro de Geografia e Estatística ( IBGE). Ou seja, o período de 56 horas de registros tem grande representatividade em relação à distribuição espacial dos tweets produzidos no Rio de Janeiro. Setores censitários foram adotados como a fonte de dados mais detalhada e estatisticamente significativa disponível no contexto brasileiro em relação aos dados de renda que necessitaríamos adiante.

$\mathrm{O}$ segundo passo focou no reconhecimento de usuários do Twitter úteis para nosso estudo. Antes de tudo, buscamos diferenciar usuários humanos de usuários automatizados (twitter bots), que postam um alto número de tweets por razões comerciais. Os usuários com um número alto de tweets de uma mesma posição foram avaliados caso a caso. Dada a distribuição da cauda longa do número de tweets por usuário, esses são um pequeno número. Usuários identificados como bots foram excluídos do banco de dados. Em seguida, precisávamos manter apenas os usuários que postassem tweets de diferentes posições no espaço, de modo a fornecer informações para a análise de trajetórias no espaço urbano. Mantivemos apenas os usuários que tweetaram pelo menos três vezes de diferentes setores censitários no período. Dos 20.192 usuários iniciais, esse procedimento levou a 2.543 
usuários, cujos 20.029 tweets poderiam gerar trajetórias espaciais dentro da rede urbana.

O passo 3 identificou a localização residencial provável dos usuários. Essencialmente, precisávamos inferir a localização residencial para inferir suas trajetórias prováveis. Avaliamos a repetição da localização do primeiro tweet da manhã (a partir das 5am) durante o período de observação. Optamos pelo primeiro tweet da manhã, porque tweets produzidos durante a noite trazem limitações para a amostra quanto ao comportamento dos usuários (Longley et al., 2015). Como a amostra já havia passado por filtros anteriores, a localização residencial pôde ser inferida para todos os 2.543 usuários.

$\mathrm{O}$ passo 4 identificou os caminhos mais curtos entre as posições dos tweets dos usuários dentro da rede de ruas do Rio de Janeiro. A geração dos caminhos entre localizações dos tweets é baseada na medida topológica de betweenness centrality (intermediação), também de autoria de Freeman (1978). Considerando a relação entre a localização do tweet (geostamp) e a rede real de ruas, nosso método fez uso de software Geographic Information System (GIS) para georeferenciar cada tweet dentro de uma precisão de $10 \mathrm{~m}$. Atribuímos uma sequência numérica para os tweets de cada usuário, de acordo com o timestamp dos tweets. Os primeiros tweets identificados foram tomados como origem das trajetórias. Conectamos as posições dos tweets postados sequencialmente durante o período sobre o sistema viário do Rio de Janeiro. A geração de caminhos mais curtos é amplamente utilizada como preditora de rotas reais (veja Bovy, 2009; Hillier et al., 1993). Os caminhos foram gerados via algoritmo de Dijkstra (1959) e o Open Street Maps (OSM).

No passo 5 , diferenciamos os usuários do Twitter de acordo com a renda per capita. Para tanto, analisamos a distribuição da renda per capita e atribuímos níveis de renda aos usuários através de um procedimento que exigiu o cruzamento de suas localizações residenciais com dados econômicos coletados naqueles setores. Uma vez que inferimos a localização residencial, atribuímos a renda média daquele setor aos usuários. $\mathrm{O}$ procedimento de inferir a renda dos usuários individuais a partir da média de residentes de cada setor requer atenção especial, já que envolve riscos de falácia ecológica envolvidos. Foi necessária uma análise de sensibilidade sobre a heterogeneidade da renda dentro dos blocos do censo. Avaliamos estatisticamente esse risco verificando o Coeficiente de Variação da renda (CV) dentro de cada setor. Dentro da cidade do Rio de Janeiro, a unidade do setor tem um número médio de 210 famílias e 616 habitantes e uma área média de $33.017 \mathrm{~m}^{2}$ (uma variação considerável é encontrada na área). A unidade do setor censitário foi a menor disponível. Vimos que a variação média da renda dentro de cada setor censitário no Rio é baixa, cerca de 9,3\%. Portanto, podemos dizer que há homogeneidade suficiente dos valores de renda dos residentes no mesmo setor para usarmos a renda média no setor como uma proxy da renda individual dos usuários do Twitter.

$\mathrm{O}$ resultado foi termos 2.543 usuários divididos em cinco grupos de renda. Analisamos a distribuição de renda aplicando uma classificação padrão de renda no Brasil proposta por Neri (2010) com base no potencial de consumo, gerando os seguintes níveis: até R\$ 750; de R \$750,01 a R\$ 1600; de $\mathrm{R} \$ 1600,01$ a $\mathrm{R} \$ 2500$; de $\mathrm{R} \$ 2500,01$ a $\mathrm{R} \$ 3400$; e R\$ 3400,01 acima. Estes valores foram identificados como faixas baixa, média-baixa, média, média-alta e alta renda (diferenciadas por cores na Figura 3).

Usamos um teste estatístico para avaliar o quão representativos são os usuários do Twitter em relação à população real do Rio de Janeiro. De fato, os dados dos rendimentos reais coletados da população em geral estão contidos nos níveis de renda atribuídos aos usuários através do nosso procedimento. No entanto, o principal risco neste estágio era a possibilidade de usuários do Twitter tenderem a ter rendas mais altas do que a população geral e a viver em setores censitários mais ricos. Isso implicaria uma amostra com menor proporção de pessoas de baixa renda, levando a uma distribuição de renda completamente diferente, longe de uma imagem razoável do cenário de renda do Rio. Assim, comparamos o histograma de renda 

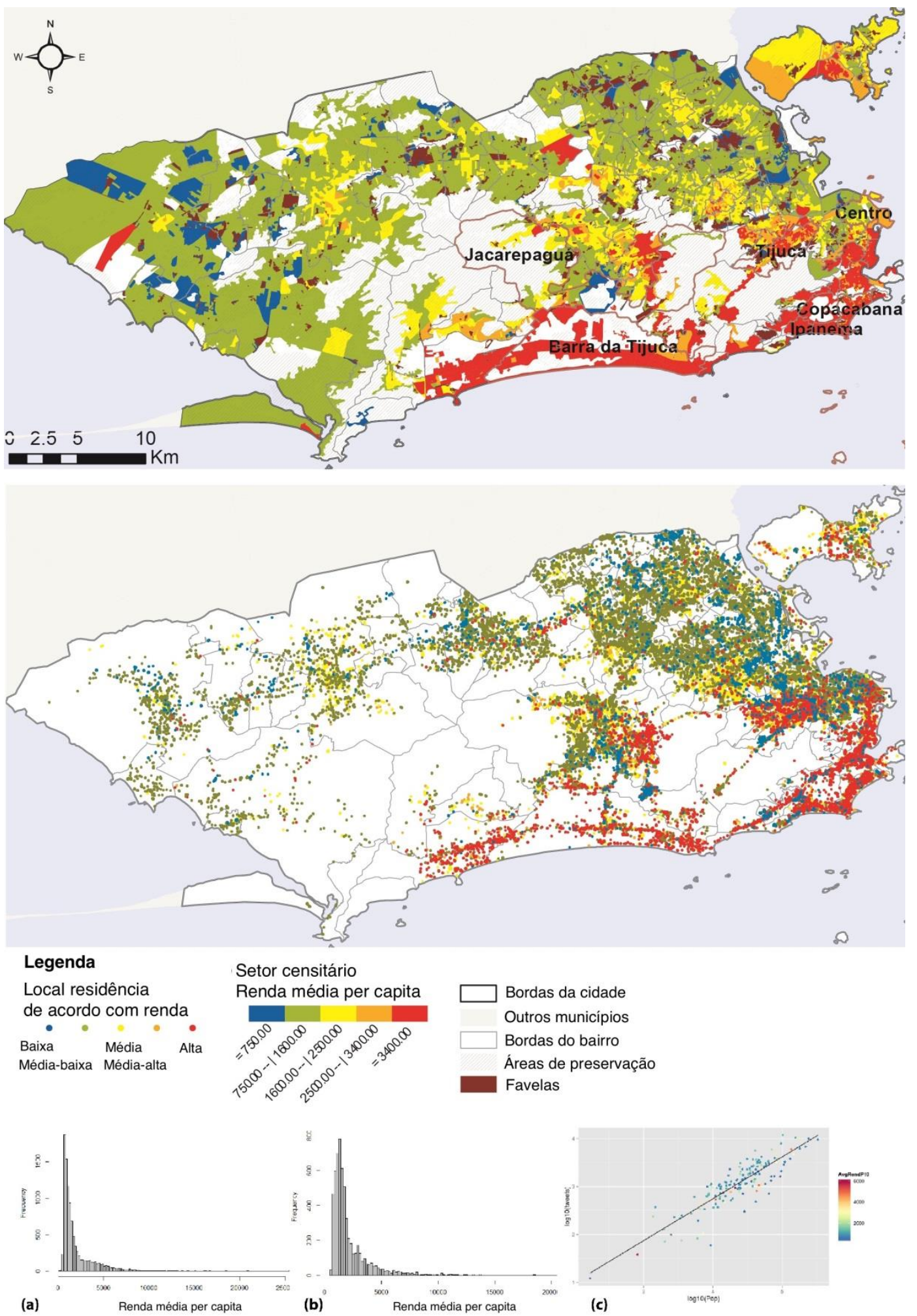

Figura 3. Níveis de renda nos setores censitários (azul ao vermelho, acima) e localizações estimadas dos usuários do Twitter (abaixo). Nos gráficos mais abaixo, temos os histogramas da renda média per capita na população do Rio (a) e dos usuários do Twitter (b). Já o gráfico (c) mostra a regressão entre as distribuições dos usuários (Y) e da população $(X)$ nos setores censitários. As cores dos pontos nesse gráfico se referem à variação da renda média nos setores, de baixa (azul) à alta (vermelho). 
estimada em nossa amostra com o histograma de renda da população do Rio. Como esperado, o histograma da renda da população (na Figura 3, canto inferior esquerdo) mostra uma distribuição exponencial, com uma longa cauda para valores de renda mais alta (mais de $\mathrm{R} \$$ 10.000 por mês). O mesmo limiar foi observado para a distribuição de renda estimada dos usuários do Twitter. A regressão linear entre as duas distribuições é de 0,67 ( $\mathrm{R}^{2}$ ajustado), mostrando que a distribuição de renda dos usuários tem um grau razoável de semelhança à distribuição de renda da população em geral (parte inferior da Figura 3, à direita). Isto sugere que o uso do Twitter não está associado a níveis de renda mais altos, confirmando achados anteriores sobre a alta taxa de penetração do Twitter no Brasil (Graham e Stephens, 2012). A análise geográfica na Figura 3 mostra a localização residencial da população com diferentes níveis de renda (no alto) e a localização dos usuários (abaixo).

\section{Encontros no espaço e no tempo: um experimento digital}

O que este experimento digital mostra sobre a dinâmica do encontro potencial entre os socialmente diferentes? Contamos o número e extensão dos trajetos dos usuários do Twitter classificados pelo nível de renda em cada segmento de rua onde houve passagem de usuários. Esta informação foi registrada para cada usuário e acumulado em sua respectiva faixa de renda. Em seguida, calculamos a sobreposição de trajetos dos grupos de renda, usando o número de atores de cada grupo passando por cada segmento de rua. Os mapas na Figura 4 mostram o grupo de renda dominante nas ruas que compõem suas trajetórias. O critério para determinar visualmente a presença dominante de um grupo ao longo de um segmento de rua é 'o grupo com proporcionalmente o maior número de caminhos sobrepostos em um segmento de rua determina a cor para esse segmento'. Uma vez que consideramos a proporção de grupos de renda em números reais, quando um grupo tem um ou mais usuários acima desse percentual, tem presença dominante.
A primeira coisa que podemos perceber é a forte influência da 'segregação residencial' relacionada com a renda. Os mais pobres tendem a morar mais ao norte e oeste da cidade, e se espalham mais amplamente sobre a paisagem urbana. Grupos de renda baixa e média-baixa mostram mais sobreposição. As amenidades paisagísticas relacionadas com a proximidade ao mar, a sul, são um fator na definição de valores da terra e localização de pessoas de maior renda no Rio. O relevo aumenta consideravelmente a complexidade dos padrões de localização residencial no Rio, ao incluir habitação em áreas não originalmente destinadas para esse fim. As favelas espalhadas no território permitem que pessoas de menor renda também vivam perto do mar e do centro de empregos no extremo leste (Central Business District / CBD). Essa paisagem urbana incomum enreda as trajetórias, como vemos em áreas da zona sul do Rio, aumentando o potencial de encontros entre os diferentes. Em segundo lugar, os resultados da análise mostram vestígios de uma segregação 'dinâmica' na forma de caminhos apropriados exclusivamente por grupos de renda específicos. Complexidades consideradas, temos um padrão geral, com os usuários de renda mais alta movimentando-se sobretudo na zona sul e sudoeste, perto do mar. Uma mudança gradual nos níveis de renda dos usuários é visível nos trajetos que se distribuem para norte e oeste.

Mas onde estão os espaços compartilhados pelos diferentes grupos sociais? Grupos de menor renda (GR1 x GR2) compartilham muito mais espaços entre si, principalmente no norte e oeste do Rio (Figura 5, à esquerda). Já a sobreposição entre os mais pobres e os ricos (GR1 x GR5) mostra baixíssimo potencial de contato (Figura 5, à direita).

Essas sobreposições visuais entre grupos de renda podem também ser avaliadas quantitativamente, assim como o quão isolada é a presença de um único grupo nas ruas e a proporção dos grupos de renda nos caminhos compartilhados (Tabela 1). Os grupos de renda menor (GR1 e GR2) são mais segregados em seus movimentos na cidade, respetivamente com $19,2 \%$ e $29,9 \%$ de suas trajetórias ocorrendo em ruas não compartilhadas. Seus trajetos também 

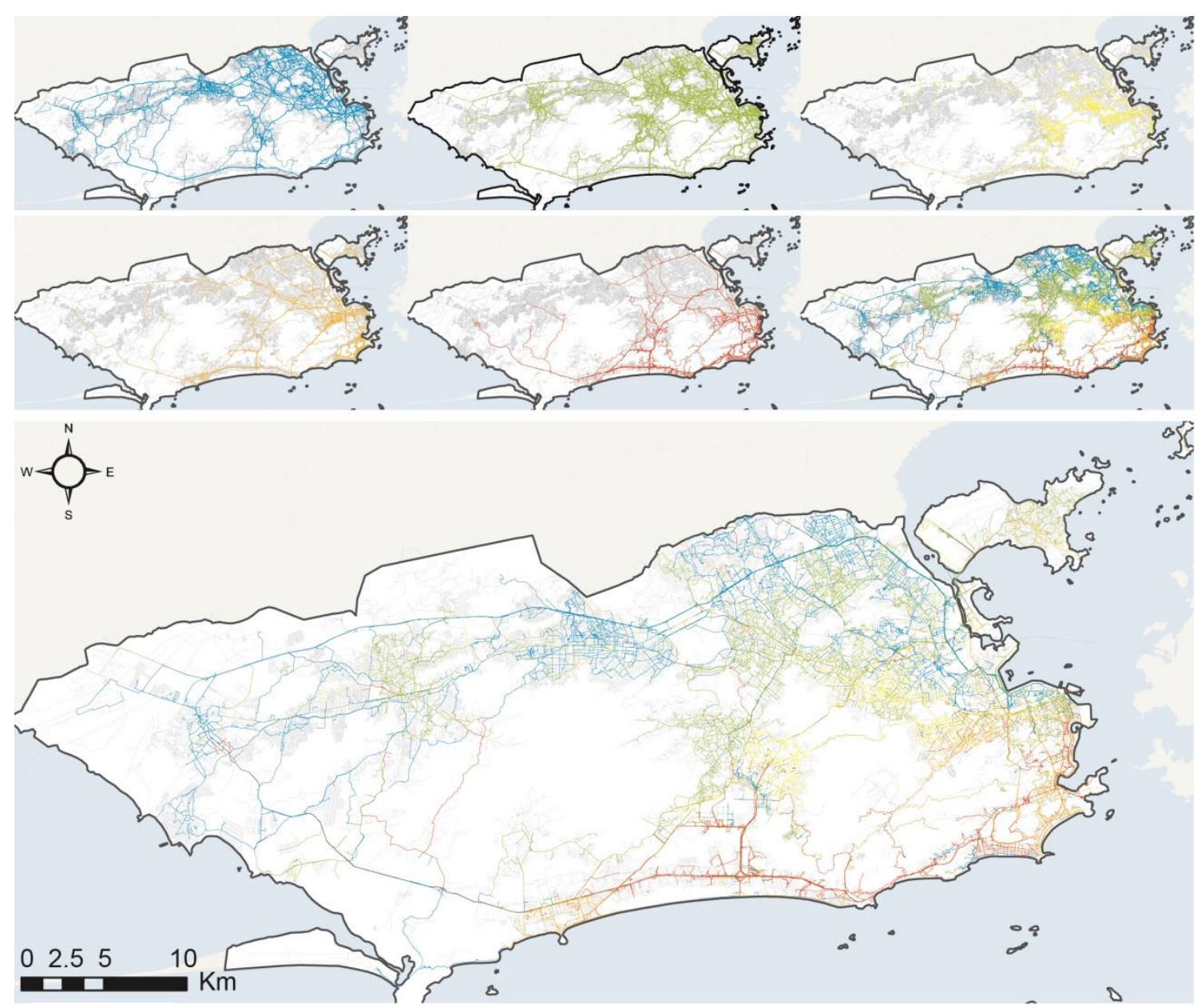

Legenda Grupo de renda predominante

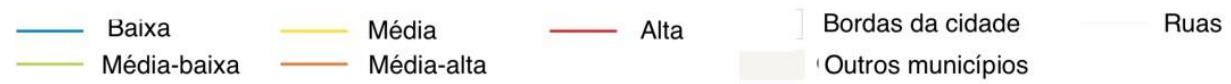

Figura 4. Redes segregadas: grupos azul (renda baixa), verde (média-baixa), amarelo (média), laranja (média-alta) e vermelho (alta). O mapa maior mostra a rede de classe dominante.

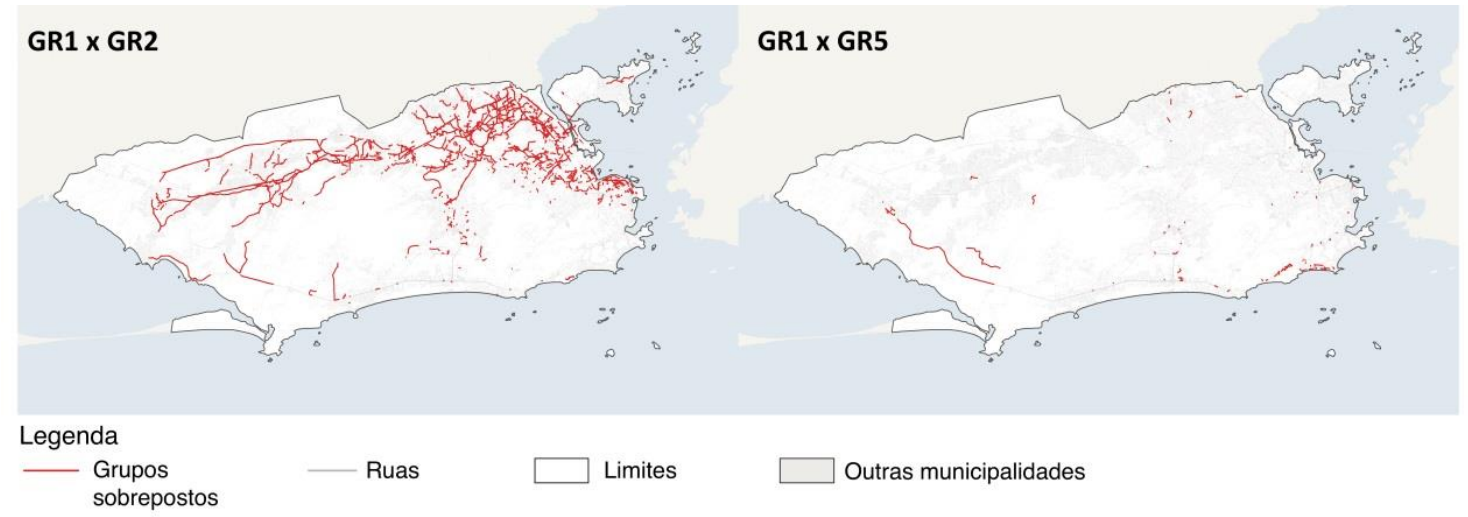

Figura 5. Potencial contato entre grupos de renda baixa (GR1xGR2) e grupos de baixa e alta rendas (GR1xGR5). 
Tabela 1. Matriz de proporção de ruas (em relação ao total de ruas) apropriadas exclusivamente por um grupo de renda individual (itálico), e a proporção de ruas compartilhadas por diferentes grupos de renda.

\begin{tabular}{lccccc}
\hline & GR1 & GR2 & GR3 & GR4 & GR5 \\
\hline GR1 & $19.2 \%$ & $10.4 \%$ & $0.5 \%$ & $0.5 \%$ & $0.8 \%$ \\
GR2 & & $29.9 \%$ & $1.5 \%$ & $1.7 \%$ & $1.2 \%$ \\
GR3 & & & $4.3 \%$ & $0.2 \%$ & $0.3 \%$ \\
GR4 & & & & $4.3 \%$ & $0.7 \%$ \\
GR5 & & & & & $4.6 \%$ \\
\hline
\end{tabular}

mostram menos diversidade social: eles são facilmente o grupo dominante (isto é, sua presença está acima da sua proporção no número total de atores). Eles compartilham mais espaços entre si que outros pares de grupos $(10,4 \%)$. O GR2 mostra um comportamento espacial menos segregado mas também tem uma participação maior de usuários $(46,7 \%)$. O fato de que o GR1 consiste em 23,6\% do total dos usuários e são dominantes em 30,5\% das ruas por onde passam sugere que eles são mais segregados do que os outros grupos em seus movimentos. Finalmente, os mais pobres e os mais ricos (GR1 e GR5) compartilham apenas $0,8 \%$ dos seus caminhos.

Considerando agora a relação entre segregação 'residencial' e segregação 'dinâmica', aquela que se manifesta sobre as pessoas e suas trajetórias (Netto e Krafta, 1999; Netto et al., 2015), o quanto diferentes grupos de renda passam por áreas residencialmente segregadas? Avaliamos essa relação cruzando a renda média nos setores censitários com a renda média do grupo dominante passando nessas áreas (Tabela 2). Mesmo que possam estar presentes em setores mais ricos, os grupos mais pobres (GR1 e GR2) são fortemente concentrados nas áreas mais pobres: 72,1\% das trajetórias GR1 acontecem em setores de renda baixa-média (S2). Os usuários mais ricos (GR4 e GR5) tendem a se mover mais nos seus próprios setores de origem (S5): $58,58 \%$ dos trajetos do GR5 acontecem em áreas S5. Por sua vez, os setores de renda média e média-alta (S3 e S4) estão abertos a grupos de renda mais diversos. O grupo de renda média (GR3) se mostra o mais distribuído em diferentes setores ao longo de seus movimentos na cidade.

Onde os diferentes grupos de renda convergem mais intensamente? Quais são as ruas com mais diversidade social? Mensuramos a diversidade social nas ruas, examinando o nível de sobreposição de trajetórias. Para tanto, usamos a fórmula da entropia da informação de Shannon (1948) para calcular a participação de cada classe sobre o número total de atores em cada segmento de rua. Os espaços com a presença de todos os grupos de renda em proporções iguais contêm a diversidade mais alta. Associamos os níveis de diversidade com cores diferentes de cinza para vermelho (Figura 6). A diversidade foi calculada para cada segmento de rua. Intervalos de proporção entre rendas foram definidos estatisticamente através de natural breaks.

$$
\text { Diversidade }=-\sum_{i} P_{i} \log _{2}\left(P_{i} / P t\right)
$$

onde Pi é o número total de usuários com renda e Pt é o número total de usuários passando em cada um dos segmentos da rua

A Figura 6 mostra uma rede de ruas de alta acessibilidade capazes de convergir diferentes grupos de renda - uma sobreposição de trajetos ao redor da zona sul (Copacabana e Ipanema) e do centro de empregos (mais a leste). Espaços de convergência social são mais encontrados nessas áreas, ou em centralidades como a Tijuca e Jacarepaguá, um pouco mais ao norte.

A configuração urbana é ativa nesses trajetos convergentes. Isso é inerente à própria modelagem do problema. A geração dos caminhos entre as localizações dos tweets é baseada na acessibilidade interna ao sistema viário a partir dos caminhos mais curtos entre segmentos de rua, apresentada no passo 4 do método. Dado que a seleção de usuários do Twitter foi aleatória, sua distribuição residencial é representativa da distribuição da população do Rio e seus tweets tendem a se distribuir pela estrutura urbana, os trajetos entre tweets emergem da 
Tabela 2. Proporção de presença de grupos de renda (GR) em setores residenciais (S), considerando o rendimento médio nos setores. Os números indicam o grupo de renda, da mais baixa (1) à mais alta (5).

\begin{tabular}{cccccc}
\hline & \multicolumn{5}{c}{ Presença dominante do Grupo de Renda (GR) } \\
& GR1 & GR2 & GR3 & GR4 & GR5 \\
\hline S1 & $9,1 \%$ & $3,9 \%$ & $3,6 \%$ & $1,7 \%$ & $5,2 \%$ \\
S2 & $72,1 \%$ & $52,4 \%$ & $28,0 \%$ & $18,2 \%$ & $17,2 \%$ \\
S3 & $13,5 \%$ & $30,1 \%$ & $29,3 \%$ & $15,9 \%$ & $7,4 \%$ \\
S4 & $2,0 \%$ & $8,4 \%$ & $20,5 \%$ & $17,5 \%$ & $11,4 \%$ \\
S5 & $3,3 \%$ & $5,2 \%$ & $18,7 \%$ & $46,7 \%$ & $58,8 \%$ \\
\hline & $100 \%$ & $100 \%$ & $100 \%$ & $100 \%$ & $100 \%$ \\
\hline
\end{tabular}

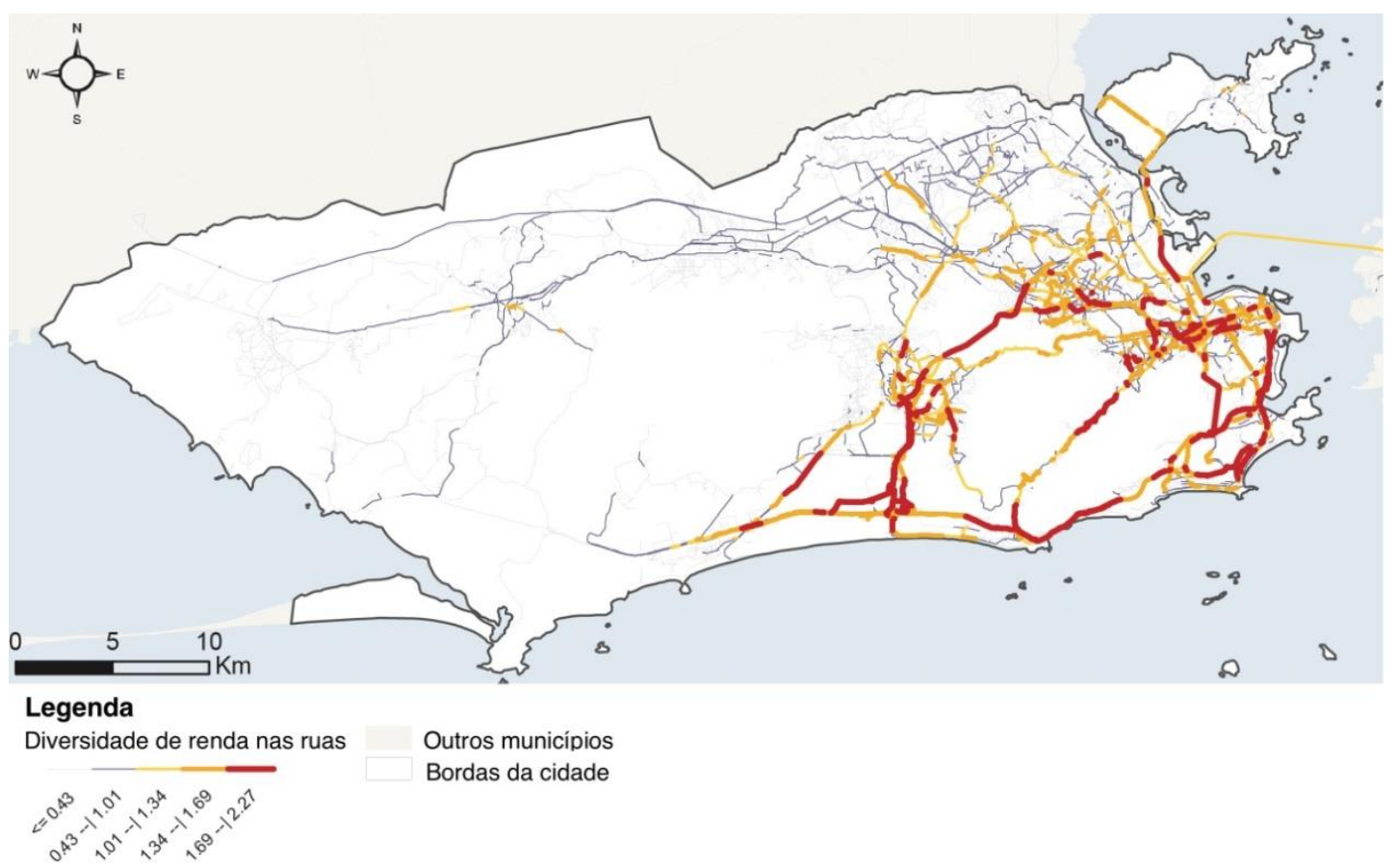

Figura 6. O mapa mostra em vermelho os espaços com maior diversidade social.

relação entrepartes que constitui essa estrutura. $\mathrm{O}$ padrão de ruas com mais diversidade social que emerge na Figura 6 se sobrepõe largamente aos caminhos mais curtos entre todas as ruas da cidade.

Podemos chegar agora à condição 'temporal' dos encontros potenciais entre os usuários do Twitter. Usando a rede OSM de ruas e os tweets com tempo e geolocalização para reconstruir os caminhos mais curtos entre tweets, nós temporalizamos essas trajetórias, calculando a velocidade média dos usuários entre os lugares a partir da indicação de tempo de cada tweet.
Consideramos os caminhos que levaram às localizações dos tweets. Por isso, mesmo que os usuários tenham postado tweets de dentro de edifícios, os caminhos entre essas localizações é que foram considerados como campo de visibilidade e encontro potencial. Por simplicidade, este campo foi definido como o segmento de rua, próximo à definição de isovistas (Benedikt, 1979). Edifícios contêm partições que não permitem campos de visibilidade longos como os segmentos de rua enquanto unidades de espaço público. Inferimos o encontro potencial como o cruzamento de trajetos dentro de um 
segmento de rua e dentro de uma janela temporal de cinco minutos. Esta janela temporal é uma definição arbitrária, capaz de incluir a quantidade de tempo para que uma pessoa apareça no campo visual de outra enquanto se desloca no espaço público. É ampla o bastante para absorver incertezas inerentes ao método em relação às trajetórias e velocidades estimadas dos usuários. Em suma, computamos as situações de encontro potencial em que usuários passaram por um mesmo segmento de rua dentro de um intervalo de cinco minutos.

Finalmente, temos condição de avaliar a sequência 'espaço-temporal dos encontros potenciais' usando uma forma de representação gráfica inspirada na geografia temporal de Hägerstrand (Figura 7). A análise mostra que o número de encontros potenciais tem um pico no início da manhã, outro por volta das $12 \mathrm{~h}$, e outro por volta das $17 \mathrm{~h}$, caindo à noite, quando os atores tendem a se encontrar em localizações residenciais (Figura 7, gráfico no canto inferior esquerdo). Concentram-se especialmente em torno dos principais canais de acessibilidade entre as áreas predominantemente residenciais e o centro do Rio, o principal polo de empregos, a leste, junto à baía de Guanabara. Ainda se distribuem no entorno dessas vias, em centralidades locais, bastante visíveis em Jacarepaguá e Zona Norte. Os pontos no mapa variando de azul para vermelho indicam agrupamentos de encontros potenciais (o número de encontros é normalizado entre 0 e 1 , este para posições de maior concentração). A fim de entender o padrão espacial dos encontros potenciais, aplicamos a função K de Ripley para resumir as dependências espaciais de forma interativa, como agrupamento ou dispersão de encontros sobre uma gama de distâncias selecionadas aleatoriamente. Como em Getis e Ord (1992), a função-K foi calculada como:

$$
L(d)=\sqrt{\frac{A \sum_{i=1}^{n} \sum_{j=1 . j \neq 1}^{n} k_{i, j}}{\pi \times n(n-1)}}
$$

onde

$\mathrm{d}=$ distância entre os locais de encontros potenciais

$\mathrm{n}=$ número total de locais de encontros potenciais
$\mathrm{A}=$ área total compreendida por encontros potenciais

$\mathrm{k}_{-}(\mathrm{i}, \mathrm{j}$ ) = peso (número de encontros potenciais em cada lugar).

O total de encontros estimados entre usuários do Twitter selecionados no Rio de Janeiro é mostrado na linha vermelha, no gráfico à direita na Figura 7 (canto inferior direito). A linha azul no gráfico mostra a função $\mathrm{K}$, indicando a distância média entre lugares de encontro selecionados aleatoriamente entre os milhares de encontros analisados. $\mathrm{O}$ eixo $\mathrm{X}$ representa a distância entre os lugares de encontro. $\mathrm{O}$ eixo $\mathrm{Y}$ representa a distância média entre os lugares de encontro ponderada pelo número de encontros. A diferença entre as linhas indica que os encontros potenciais observados estão agrupados. Como a curva do $\mathrm{K}$ observado está acima do chamado 'envelope de confiança', ela é estatisticamente significativa.

$O$ que este padrão de sobreposição implica quanto aos 'encontros potenciais' entre usuários de diferentes níveis de renda? Como avaliar mais precisamente os efeitos de diferentes rendas e trajetos espaço-temporais sobre as oportunidades de encontro? Para avaliar esses efeitos, primeiro exploramos uma distribuição de renda com mais níveis (os círculos em tons de cinza na Figura 8) e calculamos o número total de encontros potenciais entre grupos de diferentes níveis de renda. Então geramos uma análise mais convencional de redes sociais entre os usuários agrupados de acordo com sua renda. Usamos o algoritmo ForceAtlas2 (Jacomy et al., 2014) para calcular relacionamentos como um grafo. $\mathrm{O}$ grafo resultante é uma 'rede de proximidade' que relaciona grupos de renda com base em encontros potenciais. Nesta representação, os vértices representam grupos de usuários dentro de um mesmo nível de renda. As ligações mostram o número de encontros potenciais entre grupos de usuários de mesma renda (vértices na Figura 8). Quanto maior o número de encontros potenciais entre grupos, mais curta e espessa a ligação. A Figura 8 mostra que grupos de usuários mais pobres têm maior probabilidade de ter contato entre si; da mesma forma, os usuários com maior renda são menos propensos a ter contato com 

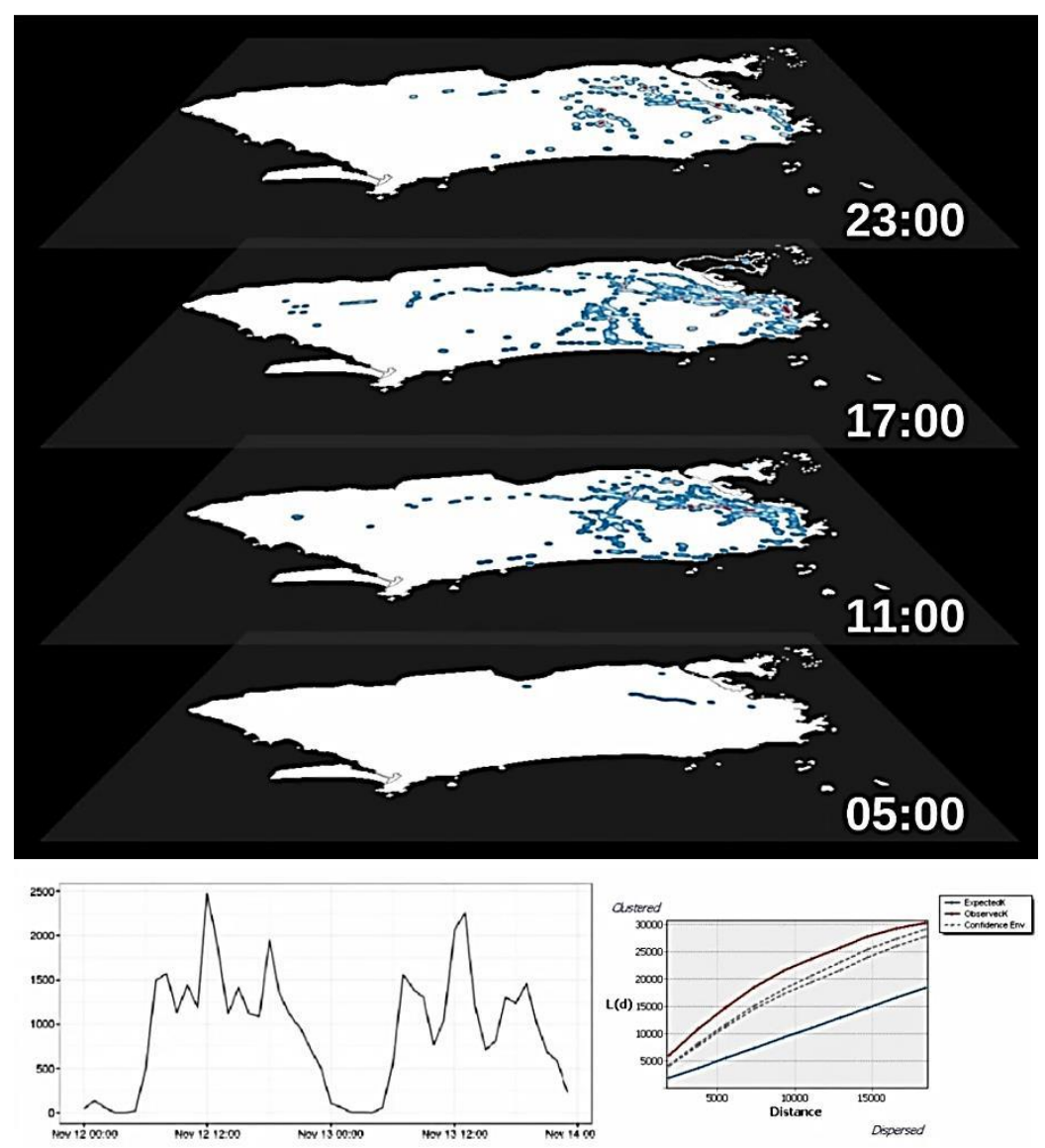

Figura 7. Prisma espaço-temporal para a intensidade dos encontros inferidos entre usuários do Twitter no Rio (acima). Número de possíveis encontros no tempo (gráfico inferior à esquerda) e uma análise do seu agrupamento no espaço (função $K$, gráfico inferior à direita).

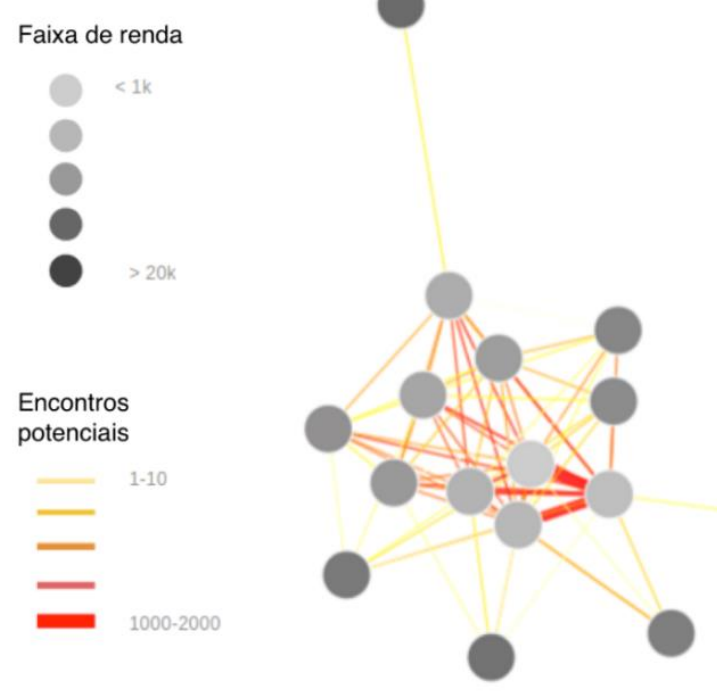

Figura 8. Redes de proximidade baseada na intensidade de encontros entre grupos de usuários de diferentes níveis de renda. 
usuários mais pobres. Os encontros mais prováveis ficam evidenciados como sendo entre pessoas socialmente 'semelhantes'.

\section{Conclusão: espaço, tempo e segregação na geografia do encontro}

Neste trabalho, exploramos o papel das trajetórias urbanas na criação de oportunidades de encontro - e seu oposto, na disjunção de encontros em uma forma de 'segregação em tempo real'. Fizemos isso explorando dados de localização das redes sociais para compreender as trajetórias dos usuários do Twitter e inferir encontros potenciais entre eles. Aplicamos essa abordagem em um estudo empírico incluindo pessoas com diferentes níveis de renda no Rio de Janeiro. Avaliamos níveis de segregação e diversidade social nas ruas e desenvolvemos uma análise de 'redes de proximidade' baseadas em encontros potenciais.

Nossa abordagem oferece uma mudança do foco das redes sociais centradas em atores para as redes de proximidade geradas a partir de uma 'geografia temporal do encontro'. Nossa hipótese foi que a probabilidade de encontros entre grupos em larga escala inclui, mas vai além da localização residencial e da segregação espacial. Seria moldado pela renda, pela distribuição de atividades, padrões de acessibilidade e pelas trajetórias das pessoas. Nossa análise das sequências espaciais e temporais de encontros potenciais nos permitiu trazer à tona uma face sutil de segregação como 'disjunção de encontros', perto da definição seminal de Freeman (1978) de segregação como 'restrições do contato'. Nesse estudo, os caminhos inferidos dos usuários do Twitter mostram maior superposição entre aqueles socialmente similares.

Naturalmente, uma série de perguntas podem ser feitas: trajetórias pouco sobrepostas podem ser interpretadas como segregadas? Seria 'compartilhar espaço' o suficiente para produzir integração social? Ao contrário da maioria dos trabalhos disponíveis na literatura, nossa abordagem é voltada para rastrear o movimento das pessoas, relacioná-lo com padrões de diferenciação social (neste caso, baseada na renda), e avaliar o seu papel nas trajetórias inferidas de pessoas no espaço urbano. Ela mostra que padrões de localização residencial e de trajetórias divergentes levam a menos oportunidades de encontro, como vimos entre os usuários mais ricos e os mais pobres do Twitter. Se Freeman (1978) tem razão ao afirmar que a segregação opera através de restrições do contato, a falta de espaços públicos compartilhados é uma parte essencial da 'experiência' e da 'reprodução' da segregação.

Um estudo baseado em dados do Twitter seria suficiente para chegarmos a esse cenário? Este experimento não está sujeito à validação dos achados, uma vez que isso exigiria acesso pessoal aos usuários para obter informações sobre seus níveis reais de renda, seus modos de transporte e suas trajetórias reais na cidade, itens que não podem ser incluídos devido ao anonimato dos usuários do Twitter e por envolverem outros recursos de pesquisa - muito mais custosos. Devido à dificuldade de generalizar conclusões a partir de amostras de usuários, procedimentos de atribuição de localização e de renda aos usuários devem ser vistos como uma aproximação em vez de um cenário real, como insistimos. Como uma proxy para o cenário de encontro e segregação potenciais, este experimento com base em dados de localização do Twitter pode mostrar apenas 'tendências' nas trajetórias de um grande número de atores. Ainda assim, os resultados encontrados encontram fortes convergências com estudos empíricos conduzidos no Rio de Janeiro a partir de entrevistas com 240 pessoas (Netto e Furtado, 2017), e em Niterói, com 121 pessoas (Netto et al., 2015) de rendas distintas. É interessante perceber que, mesmo que forneçam confiabilidade na medida em que entrevistados forneçam corretamente suas rendas e trajetórias, estes dois estudos não oferecem uma 'geografia temporal dos encontros', pela imprecisão do dado de tempo associado às atividades e percursos dos entrevistados.

Por sua vez, o presente estudo sugere que os dados oferecidos pelo Twitter são um meio para identificar padrões de movimentação de atores, abrindo fortes possibilidades para a compreensão de condições de reprodução da integração e segregação social. Os dados do Twitter não 
permitem inferir trajetos completamente mas as análises gráficas e quantitativas da sobreposição dos trajetos que inferimos oferecem possibilidades de compreensão da segregação para além dos mapas estáticos de localização residencial ou de atividade. Este é um dos propósitos da nossa abordagem: nos aproximarmos de uma leitura das redes segregadas de movimentação - assim como dos espaços públicos com diferentes potenciais de sobreposição. Esses movimentos segregados e o potencial para a sobreposição de redes sociais não podem ser inferidos somente a partir da renda, atividade ou distribuição residencial.

As mídias digitais oferecem uma possibilidade extraordinária de pesquisa: são uma fonte para entendermos a geografia elusiva dos encontros de milhares de pessoas, aqui introduzida, no melhor do nosso conhecimento, pela primeira vez na literatura. Isso parecia praticamente impossível antes dos dados locacionais oferecidos em tempo real por usuários de redes digitais e dispositivos móveis de comunicação. Nossa abordagem sugere que a probabilidade de encontro está impregnada de espacialidade, interagindo ativamente com a configuração urbana para gerar potenciais de convergência e copresença de grupos sociais. As chances de encontrar 'o outro' se mostram distribuídas de acordo com essa estrutura espacial e temporal da ação dentro na cidade.

\section{Referências}

Allen, T. (1977) Managing the flow of technology (MIT Press, Cambridge).

Batty, M. (2013) The new science of cities (The MIT Press, Cambridge).

Batty, M. (2017) 'Endorsement', em Netto, V. The social fabric of cities (Routledge, Londres).

Benedikt, M. L. (1979) 'To take hold of space: isovists and isovist fields', Environment and Planning B: Planning and Design 6, 47-65.

Bettencourt, L. M. A. (2013) 'The origins of scaling in cities', Science 340, 1348-441.

Boettcher, A. e Lee D. (2012) 'EventRadar: a real-time local event detection scheme using Twitter stream', Proceedings of the IEEE International Conference on Green Computing and Communications, 358-67.

Bourdieu, P. (1985) 'The social space and the genesis of groups', Theory and Society 14, 72344.

Bovy, P. H. L. (2009) 'On modelling route choice sets in transportation networks: a synthesis', Transport Reviews 1, 43-68.

Dijkstra, E. W. (1959) 'A note on two problems of connexion with graphs', Numerische Mathematik 1, 269-71.

Fischer C. e Shavit Y. (1995) 'National differences in network density: Israel and the United States', Social Networks 17, 129-45.

Freeman, L. (1978) 'Segregation in social networks', Sociological Methods and Research 6, 411-29.

Getis, A. e Ord, J. K. (1992) 'The analysis of spatial association by use of distance statistics', Geographical Analysis 24, 189-206.

Giddens, A. (1984) The constitution of society (Polity Press, Cambridge).

Goffman, E. (1961) Encounters (Bobbs-Merrill, Indianapolis).

Gonzales, M., Hidalgo, C. e Barabási, A-L (2008) 'Understanding individual human mobility patterns', Nature 453, 479-82.

Graham, M, Stephens, M. (2012) 'A geography of Twitter' (www.oii.ox.ac.uk/vis/?id=4fe09570) consultado em 9 de Setembro de 2017.

Hägerstrand, T. (1970) 'What about people in regional science?', Papers of the Regional Science Association 24, 6-21.

Hillier, B. e Hanson, J. (1984) The social logic of space (Cambridge University Press, Cambridge).

Hillier, B. e Vaughan, L. (2007) 'The city as one thing', Progress in Planning 67, 205-30.

Hillier, B., Penn, A., Hanson, J., Grajewski, T. e Xu, J. (1993) 'Natural movement: or configuration and attraction in urban pedestrian movement', Environment and Planning $B$ : Planning and Design 19, 38-54.

Hunt, C. L. e Walker, L. (1974) Ethnic dynamics: patterns of inter-group relations in various societies (Dorsey, Homewood).

Jacomy, M., Venturini, T., Heymann, S. e Bastian, M. (2014) 'ForceAtlas2, a continuous graph layout algorithm for handy network visualization designed for the gephi software', PLoS ONE 9, e98679.

Kwan, M-P. (2013) 'Beyond space (as we knew it): toward temporally integrated geographies of segregation, health, and accessibility', Annals of the Association of American Geographers 103, 1078-86.

Lee, J. e Kwan, M. P. (2011) 'Visualisation of socio-spatial isolation based on human activity patterns and social networks in space-time', Tijdschrift voor Economische en Sociale Geografie 102, 468-85.

Lee, K. H., Lippman, A. e Pentland, A. (2011) 
'The impacts of just-in-time social networks on people's choices in the real world', $3^{\text {rd }}$ International Conference on Social Computing, Boston, 9 a 11 de Outubro.

Li, W., Serdyukov, P., Vries, A., Eickhoff, C. e Larson, M. (2011) 'The where in the tweet', $20^{\text {th }}$ ACM International Conference on Information and Knowledge Management, Glasgow, 24 a 28 de Outubro.

Longley, P., Adnan, M. e Lansley, G. (2015) 'The geotemporal demographics of Twitter usage', Environment and Planning A 47, 465-84.

Marques, E. C. (2012) 'Social networks, segregation and poverty in São Paulo', International Journal of Urban and Regional Research 36, 958-79.

Neri, M. C. (2010) A nova classe média: o lado brilhante dos pobres (FGV, Rio de Janeiro).

Netto, V. M. (2014) Cidade \& sociedade: as tramas da prática e seus espaços (Editora Sulina, Porto Alegre).

Netto, V. M. e Furtado, M. (2017) 'Configuration, location, mobility: effects on spatial and social behaviour for residents in social housing complexes in Rio de Janeiro, Brazil', Proceedings of the $11^{\text {th }}$ International Space Syntax Symposium, (Instituto Superior Técnico, Lisboa) 96.1-14.

Netto, V. M. e Krafta, R. (1999) 'Segregação dinâmica urbana: modelagem e mensuração', Revista Brasileira de Estudos Urbanos $e$ Regionais 1, 133-52.

Netto, V. M. e Krafta, R. (2001) 'Socio-spatial networks: social segregation as a real-time phenomenon', em Peponis, J, Wineman, J e Bafna, S. (eds.) Proceedings of the $3^{\text {rd }}$ International Space Syntax Symposium (Georgia Institute of Technology, Atlanta) 34.1-10.

Netto, V. M, Pinheiro, M. e Paschoalino, R. (2015) 'Segregated networks in the city',
International Journal of Urban and Regional Research 39, 1084-102.

Park, Y.M. e Kwan, M-P. (2017) 'Multicontextual segregation and environmental justice research: toward fine-scale spatiotemporal approaches', International Journal of Environmental Research and Public Health 14, 1205.

Ribeiro, S., Davis, C., Oliveira, D., Meira, W., Gonçalves, T. e Pappa, G. (2012) 'Traffic observatory: a system to detect and locate traffic events and conditions using Twitter', $5^{\text {th }}$ International Workshop on Location-Based Social Networks, Redondo Beach, 7 a 9 de Novembro.

Schnell, I. e Yoav, B (2001) 'The socio-spatial isolation of agents in everyday life spaces as an aspect of segregation', Annals of the Association of American Geographers 91, 62233.

Schnell I. e Yoav B. (2005) 'Globalisation and the structure of urban social space: the lesson from Tel Aviv', Urban Studies 42, 2489-510.

Selim G. (2015) 'The landscape of differences: contact and segregation in the everyday encounters', Cities 46, 16-25.

Shannon, C. E. (1948) 'A mathematical theory of communication', Bell System Technical Journal 27, 379-423.

Takhteyev, Y., Gruzd, A. e Wellman, B. (2012) 'Geography of Twitter networks', Social Networks 34, 73-81.

Veloso, A. e Ferraz, F. (2011) 'Dengue surveillance based on a computational model of spatio-temporal locality of Twitter', $3^{\text {rd }}$ International Conference on Web Science, Koblenz, 15 a 17 de Junho.

Wissink, B., Schwanenb, T., e Van Kempen, R. (2016) 'Beyond residential segregation: introduction', Cities 59, 126-30.

\title{
Tradução do título, resumo e palavras-chave
}

\section{A temporal geography of encounters}

\begin{abstract}
The integration between spatial and social networks is fundamental to new approaches on cities as systems of interaction. In this paper, we focus on the spatial and temporal conditions of encounters as a key condition for the formation of social networks. Drawing on classic approaches such as Freeman's concept of segregation as 'restriction on contact', Hägerstrand's time-geography, and recent explorations of social media locational data, we analysed the space-time structure of potential encounters latent in the urban trajectories of people with different income levels in Rio de Janeiro, Brazil. This approach allows us to estimate trajectories examining spatiotemporal positions in tweets, and assess spaces of potential encounter and levels of social diversity on the streets. Finally, we discuss the utility and limitations of an approach developed to grasp how clusters of encounters between groups with different income levels are produced.
\end{abstract}

